\title{
LOFT : a tool for assisting selection of test data sets from algebraic specifications
}

\author{
Bruno Marre \\ Université de Paris-Sud, Laboratoire de Recherche en Informatique, \\ C.N.R.S. U.R.A. 410, Bâtiment 490, F-91405 Orsay Cedex, France \\ Phone : (33) 1694166 32, Email : marre@lri.fr
}

LOFT (LOgic for Functions and Testing) is a tool allowing to implement test data selection strategies from algebraic specifications. It is the necessary complement of the software testing theory and method proposed at the L.R.I $([1,2])$. We consider positive conditional specifications : the properties of the operations are described by equational Horn clauses. For every axiom, a finite subset of its ground instances is selected by assuming "selection hypotheses" on the program under test. More precisely, it is sufficient to only consider some instances of axioms conclusions such that the premisses are valid $([1,2])$.

In this demonstration we will illustrate the implementation of the selection strategies corresponding to two commonly used selection hypotheses : the regularity and uniformity hypotheses.

A regularity hypothesis for a variable occurring in a formula makes it possible to select instances of the formula such that the "size" of the variable substitutions is bounded by some value.

A uniformity hypothesis for a variable occurring in a formula makes it possible to select one instance of the formula with any substitution for the variable. Uniformity hypotheses are generalized to set of variables belonging to a subdomain (generally of the validity domain of the formula).

In order to point out sub-domains of an axiom validity domain which are good candidates for uniformity hypotheses, we use a case analysis technique : decomposition by unfolding, i.e. "equal by equal" replacements of the operations occurring in an axiom (introducing one sub-case for each axiom defining the unfolded operation).

The selection of instances corresponding to the above selection hypotheses, as well as the unfolding strategy, can be expressed as a controlled resolution of some equational problem. Thus, the kernel of LOFT is an equational resolution procedure for positive conditional specifications (conditional narrowing). More precisely, LOFT uses an efficient simulation of narrowing through Horn clause resolution : axioms and equational goals are translated into Horn clauses and predicate goals. In order to minimize the size of a narrowing tree, simplification steps of subgoals by rewriting are applied between narrowing steps. Moreover, the strategy adopted for the choice of the occurrence to be narrowed gives priority to occurrences that unify with a minimum number of axioms. This selection rule makes it possible to detect sooner failing computations and decreases the size of a narrowing tree by giving priority to deterministic computations. The completeness of the narrowing procedure is ensured by the use of an efficient 
and complete strategy : the iterative depth first search strategy.

Two controlled resolution procedures are proposed for the implementation of the unfolding strategy. Both procedures are applied to validity domains described by conjunctions of equations. They return sub-domains expressed by pairs of substitutions and constraints (constraints are conjunction of equations involving operations that are not unfolded).

The first unfolding implementation is activated by the unfold_std command which controls the unfolding through an argument list of operations that should not be unfolded. By default, an operation can be unfolded at most one time (in order to ensure termination for recursive definitions). However, it is possible to specify an arbitrary number of unfolding steps.

The other unfolding implementation needs a more deeper understanding of the narrowing procedure. It uses do_not_unfold meta clauses specifying which pattern of equation should not be unfolded. This meta control is similar to the "wait" or "delay" directives proposed in some logic programming languages.

For each sort $s$, LOFT automatically computes a typing function is_a_s and a canonical "size" function alpha_s (inductively defined w.r.t. the sort generators). They are respectively used for implementations of uniformity selection strategies and regularity selection strategies. The "size" function of a sort computes the number of non constant generators of the sort. A regularity selection strategy for a variable $X$ of sort $s$ bounded by $b$ is implemented as the narrowing of a goal : alpha $s(X) \leq b=$ true. For the implementation of uniformity selection strategies (on a variable or on a sub-domain), we select the first solution of some goal (a typing goal for uniformity on a variable, or the equations defining the sub-domain for a uniformity on a sub-domain) computed with a random choice strategy for the choice of the axiom to be applied. This particular axiom selection rule allows to compute solutions of a narrowing goal in a non deterministic order. This strategy is activated in LOFT by the "?" command.

LOFT works on Sun4 station under X11. It was developed with the ECLIPSE prolog environment distributed by the ECRC. Its interface is programmed with the $\mathrm{Tk} / \mathrm{Tcl}$ toolkit. The demonstration of the tool is done on two examples :

- Test data are selected from a specification of sorted lists. This simple example allows to smoothly introduce selection hypotheses and strategies.

- Then, we consider a specification of the door monitoring module of an automatic subway ([3]). We show how to select test data sets allowing to verify the different alarm conditions of this monitoring module.

\section{References}

1. Bernot, G. and Gaudel, M.-C. and Marre, B. Software testing based on formal specifications: $a$ theory and a tool Software Engineering Journal, Vol.6, Num.6, pp.387405. November 1991.

2. Gaudel, M.-C. Testing can be formal, too Proceedings of TAPSOFT'95. March 1995.

3. Dauchy, P. and Gaudel, M.-C. and Marre, B. Using algebraic specifications in software testing: a case study on the software of an automatic subway Journal of Systems and Software, ELSEVIER, Vol.21, Num.3, pp.229-244. June 1993. 